\title{
Estudio diferencial del nivel de estrés percibido, liderazgo, cohesión y autoconfianza en Unidades de Élite de la Armada Española, en la Operación Active Endeavour de la DTAN
}

\author{
Galindo Ángel J. ${ }^{1}$, Galindo Ángel J. ${ }^{2}$
}

Sanid. mil. 2014; 70 (3): 174-184; ISSN: 1887-8571

\begin{abstract}
RESUMEN
Antecedentes y objetivos: Fruto de los atentados terroristas del 11 de septiembre de 2001 en Estados Unidos, surge la operación antiterrorista Active Endeavour con la participación de la Armada Española con la función de control marítimo y el abordaje de buques con actividad sospechosa terrorista. Este estudio trata de analizar las características diferenciales de los grupos especiales de abordaje de buques (boarding teams), en relación a sus niveles de estrés percibido, liderazgo, cohesión y autoconfianza. Material y métodos: Para ello se emplea una muestra de 120 militares españoles integrados en el segundo grupo marítimo de la OTAN, de los que 17 pertenecen al equipo de abordaje y el resto actuaría como grupo de comparación y control. Rellenaron el cuestionario de estrés percibido de Cohen adaptado a población española, y el Cuestionario de Perfil de Moral de Unidad que evalúa aspectos implicados en la adaptación a zona de operaciones, destacando el liderazgo, la cohesión y la autoconfianza. Resultados: Los resultados muestran que no hay diferencias estadísticamente significativas en los niveles de estrés percibido en ambos grupos, pero sí existen diferencias en el nivel de cohesión siendo mayor en el equipo de abordaje. Conclusiones: La cohesión ha sido un factor clásicamente relacionado con una mayor adaptación a entornos operativos, debido a la percepción de apoyo social que reciben los miembros del grupo, y la posibilidad de aumentar los recursos de afrontamiento frente al estrés. Los grupos de abordaje poseen un mayor nivel de exposición al riesgo debido a su función al interceptar buques sospechosos, sin embargo no presentan mayores niveles de estrés percibido, debido a la presencia de unos mejores recursos de afrontamiento. Se detecta la presencia de mayores niveles de cohesión grupal, factor clásicamente asociado a un mejor afrontamiento de situaciones estresantes. Se concluye que de todas las variables evaluadas la cohesión es especialmente alta en los grupos de abordaje, lo que puede favorecer un nivel de estrés percibido similar, aun cuando haya una mayor exposición al riesgo.
\end{abstract}

PALABRAS CLAVE: Estrés percibido, liderazgo, cohesión, autoconfianza, unidad de élite, Operación Active Endeavour.

Differential study of perceived stress level, leadership, cohesion and confidence among members in military elite units of the Spanish Navy, in NATO Operation Active Endeavour

SUMMARY: Antedecents and objectives: As a result of the terrorist attacks of September 11, 2001 in the United States, was created the antiterrorist operation Active Endeavour with the participation of the Spanish Navy. This study aims to analyze the different characteristics boarding teams, in relation to their levels of perceived stress and individual and group psychological profile they present. Material and methods: The sample consisted of 120 Spanish soldiers integrated into the second NATO maritime group, of which 17 belong to the boarding team and the rest belong to the control group. They completed the perceived stress questionnaire adapted from Cohen, and the Unit Morale Profile Questionnaire that evaluates aspects involved in adaptation to airside, emphasizing leadership, cohesion and confidence. Results: The results show no statistically significant differences in levels of perceived stress in both groups, but there are differences in the level of cohesion. Conclusions: Cohesion has been classically associated to a better adaptation to operating environments, due to the perception of social support between group members, and the possibility of increasing the resources of stress coping. The boarding teams have a higher level of risk exposure due to its role in intercepting vessels suspected; however, not have higher levels of perceived stress, because of better coping resources. The presence of higher levels of group cohesion is detected, factor associated with better coping with stressful situations. We conclude that cohesion is especially high in the boarding teams, compared to control group, what influence the result of similar level of perceived stress, even when there is a greater risk exposure.

KEY WORDS: Perceived stress level, leadership, cohesion, self-confidence, military elite units, Operation Active Endeavour.

${ }^{1}$ Cte. Psicólogo. Escuela Militar de Sanidad. Madrid. España.

${ }^{2}$ Cap. Farmacéutico. Escuela Militar de Sanidad. Madrid. España.

Dirección para correspondencia: Jorge Galindo Ángel. Escuela Militar de Sanidad. C/Camino de los Ingenieros N. ${ }^{\circ}$ 6. CP 28047. Madrid. España. jgalinan@fn.mde.es

Recibido: 17 de febrero de 2014

Aceptado: 21 de abril de 2014

\section{INTRODUCCIÓN}

Las Fuerzas Armadas Españolas vienen asumiendo la cooperación en una cada vez mayor diversidad de escenarios operativos por medio de distintos acuerdos con las Naciones Unidas, la OTAN, y el Eurocuerpo. 
En julio de 1991, se crea una Misión de Monitorización de la Comunidad Europea para el conflicto de Yugoslavia (ECCM) en la que toman parte 300 observadores desplegados en Eslovenia, Croacia y Bosnia-Herzegovina, dada la crueldad y niveles de destrucción de las acciones de ambos bandos así como el número de desplazados. El 21 de febrero de 1992, bajo el amparo de la resolución 793 del Consejo de Seguridad de la ONU (CSNU), se inicia el despliegue de una Fuerza de Protección de Naciones Unidas (UNPROFOR), formada por 22.500 efectivos de trece países, inicialmente en Croacia y, posteriormente en BosniaHerzegovina con la doble misión; (a) de vigilar el cumplimiento de los acuerdos de paz conseguidos hasta ese momento y (b) de proteger los convoyes de ayuda humanitaria.

La Armada Española tiene una importante aportación en el conflicto ${ }^{1}$, siendo ésta una de sus primeras participaciones recientes de carácter internacional. En fecha tan temprana como el 14 de julio de 1992, la fragata española Baleares se unía a la fuerza de la OTAN STANAVFORMED (Fuerza Naval Permanente en el Mediterráneo) para llevar a cabo una operación marítima en el Adriático con objeto de comprobar la efectividad de las sanciones impuestas por Naciones Unidas a Serbia y Montenegro.

Por su parte, en noviembre de 1992 se une a UNPROFOR la primera Agrupación Táctica Española (AGT) "Málaga", formada básicamente con Fuerzas del IV Tercio de la Legión, de guarnición en Ronda, con una de las tareas más duras de todo el contingente internacional; mantener abierta la ruta del río Neretva, indispensable para la circulación de los convoyes que iban a Móstar y Sarajevo desde las costas croatas. Ese mismo otoño se rompió la alianza entre croatas y musulmanes lo que derivó en una guerra total en marzo del siguiente año, suponiendo para la AGT "Canarias", relevo de la anterior, la encomienda de constituir una fuerza de interposición entre los contendientes, especialmente en la ciudad de Móstar. En tales circunstancias se produjo en mayo de 1993 la primera de las veinte bajas sufridas por nuestros soldados en territorio bosnio en un convoy que transportaba sangre y medicinas para el hospital musulmán de Móstar.

Estos hechos constituyen el inicio de la participación que las Fuerzas Armadas Españolas vienen realizando en diversos teatros de operaciones desde hace ya más de dos décadas con sus aliados. Desde entonces, han sido distintos los escenarios bélicos en los que se ha tomado parte, sufriendo éstos una considerable evolución y cambio hasta la actualidad.

Resulta esencial destacar un punto de inflexión que cambió el rumbo y el modo de proceder de las Fuerzas de Seguridad y Defensa de las distintas naciones, marcado por los atentados del 11 de septiembre de 2001 en Estados Unidos, con posteriores réplicas en España (11 de marzo de 2004), y Londres (7 de Julio de 2005). En este momento, adquiere protagonismo el riesgo de un ataque inesperado e indeterminado, y el formato de enfrentamiento asimétrico con la utilización del ataque terrorista. Con motivo de dicha amenaza, y la finalidad de controlar por vía marítima la actividad terrorista, se toman una serie de medidas tras la invocación del Artículo 5, sobre la defensa colectiva de la OTAN, por vez primera en la historia de la Alianza.

Surge así el 6 de octubre de 2001 la operación "Active Endeavour", por medio de la cual los buques de la OTAN han estado patrullando el Mediterráneo y haciendo un seguimiento del tráfico marítimo, abordando cualquier buque con actividad sospe- chosa, tratando de establecer si un buque está involucrado en actividades terroristas, evitando la proliferación y el tráfico ilícito de armas de destrucción masiva, y favoreciendo una mayor cooperación con las organizaciones no miembros de la OTAN y los organismos civiles.

La Task Force ENDEAVOUR (Fuerza desplegada para la operación) consiste en un conjunto equilibrado de unidades de superficie, submarinos y aviones de patrulla marítima. Los Grupos Marítimos 1 y 2 de la OTAN ofrecen un apoyo periódico a la Operación Active Endeavour. Según el Plan Operativo aprobado en enero de 2010, la Operación Active Endeavour está evolucionando de un sistema basado en una plataforma estable de presencia permanente a una operación basada en un sistema de información compartido por las naciones participantes, usando una combinación de unidades de activación rápida y operaciones específicas de carácter intensivo y selectivo (surge operation), favoreciendo además la cooperación con terceros países no pertenecientes a la OTAN y organizaciones internacionales, a fin de mejorar el control de la situación marítima y la preparación y el estado de alerta.

\section{El abordaje}

El abordaje constituye la acción física de control de un buque por medio de la detención del mismo, comprobación de la documentación pertinente y el registro de su carga si algo parece infrecuente o sospechoso. El equipo de abordaje lo conforma un grupo de entre 15 y 20 miembros de la dotación del buque específicamente adiestrados para ello. El nivel de hostilidad potencial del buque receptor varía situacionalmente e incluye la posibilidad de repeler o afrontar cualquier ataque esperado o inesperado.

En general son muchos los estresores con los que se enfrenta un soldado en operaciones militares (terrestres o navales). De forma genérica han sido establecidas cinco amplias dimensiones que caracterizan el espectro de posibles estresores psicológicos experimentados por los soldados en las operaciones militares actuales $^{2}$, estas son; (a) aislamiento, (b) ambigüedad del entorno, (c) impotencia, (d) hastío existencial, (e) el peligro y (f) el ritmo de la misión o navegación -OPTEMPO- ${ }^{3}$. Además, uno de los mayores estresores reconocidos por los soldados es la frecuente separación familiar ${ }^{4}$.

El abordaje supone una situación de afrontamiento de peligros reales que potencialmente generan intensos estados de ansiedad y estrés, debido a la exposición a un medio de incertidumbre donde cabe la posibilidad de un enfrentamiento con consecuencias impredecibles.

En el contexto concreto de la muestra de estudio los estresores de mayor relevancia son la presencia de peligros potenciales percibidos, o reales, donde un mando (líder) y sus subordinados se enfrentan personalmente a situaciones altamente dinámicas e impredecibles, y donde el resultado de la gestión de dichos grupos puede suponer lesiones físicas, psíquicas, o incluso la muerte de los miembros de la unidad ${ }^{5}$. De otro lado, dicho contexto se caracteriza por la presencia de una alta ambigüedad en un entorno que la mayor parte de las ocasiones resulta impredecible ${ }^{2}$. La hostilidad del buque receptor es difícil de predecir a partir de 
las comunicaciones previas que se mantienen, previo al abordaje, salvo en los casos donde la respuesta es negativa frente al mismo.

Otros estresores indirectos pero que pueden favorecer altas cotas de estrés percibido son el alto ritmo de trabajo desarrollado en el buque (OPTEMPO), en ocasiones asociado a los turnos de servicio establecidos, así como el tiempo de separación familiar motivado por la navegación, que en ocasiones se unen a los estresores específicos anteriormente detallados en dichos equipos.

En tales situaciones los soldados experimentan respuestas psicofisiológicas de estrés como una reacción normalizada de naturaleza adaptativa, cuya finalidad es la de preparar y adaptar al organismo tanto para la huida como el ataque, como medio de afrontamiento activo de una situación peligrosa que garantice la propia supervivencia. El estrés incide negativamente en los estados de salud y en la eficacia adaptativa de las personas, estando estrechamente relacionado con la cantidad de recursos de afrontamiento reales o percibidos, para afrontar las exigencias impuestas por determinadas situaciones ${ }^{6-12}$. Los distintos enfoques difieren en relación al concepto de estrés en el que se basan, siendo fundamentalmente tres las perspectivas desde las que se conceptualiza el estrés: la ambiental, la psicológica y la biológica ${ }^{13,14}$.

Lazarus y Folkman (1984) ${ }^{11}$ enfatizan en el estrés la importancia de la valoración que de la situación se hace, siendo éste el concepto de estrés percibido incorporado en la investigación en el ámbito militar. El afrontamiento de las situaciones de riesgo que potencialmente generan respuestas de estrés, dependerá según la literatura, de variables tanto de naturaleza individual como grupal.

A nivel individual, algunos autores se centran en el desarrollo de las creencias de autoeficacia de modo que la confianza en uno mismo es determinante en este tipo de contextos peligrosos ${ }^{15}$, además de la confianza en los otros miembros de la unidad. La autoeficacia es un constructo sólidamente establecido por medio de la Teoría de la autoeficacia de Bandura ${ }^{16,17}$. Es una variable asociada a otras como la confianza en sí mismo, el autoconcepto y la autoestima. En ámbitos operativos, puede ser definida como la confianza que una persona tiene en la capacidad para desarrollar de un modo eficaz las tareas encomendadas asociadas a la misión. Esta ha sido puesta a prueba por medio del análisis de la evolución de dichas creencias antes y después de duros programas de entrenamiento en paracaidistas, donde los participantes debían dominar fuertes sentimientos de exposición al riesgo y miedo. Dicha exposición incrementaba significativamente dichas creencias en estudios longitudinales ${ }^{15}$. La autoeficacia además ha sido un factor crucial asociado a la efectividad del liderazgo en distintos contextos ${ }^{18}$.

En la literatura sobre el estrés ocupacional, la autoeficacia ha sido considerada como un factor con un efecto directo y positivo con diversos parámetros de salud ${ }^{19-21}$. Los individuos con altos niveles de autoeficacia tienden a informar de mayores niveles de bienestar y unas actitudes relativas al trabajo más positivas tales como la satisfacción laboral con respecto a aquellos que presen$\tan$ baja autoeficacia ${ }^{22}$.

Además, algunos estudios sugieren que es necesario que la autoeficacia vaya asociada a la sensación de control (sobre las situaciones), para que pueda producir efectos beneficiosos o “amortiguantes" sobre el estrés ${ }^{20,21}$, percepción que resulta limitada en contextos ambiguos. De este modo, la injusticia parece tener un impacto sobre el sentido de autoestima ${ }^{23,24}$, lo que a su vez sugiere que la injusticia tenga un efecto sobre el sentido de control activo sobre el medio.

En operaciones los soldados hacen frente a gran cantidad de dificultades entre las que destacan la pérdida de sueño, altos niveles de malestar psíquico y físico, periodos prolongados de intensa vigilancia, y el afrontamiento de riesgos intensos ${ }^{25}$, estresores afrontados por los equipos de abordaje. Tales condiciones del entorno pueden incapacitar completamente a algunos solda$\operatorname{dos}^{26}$, siendo el estrés un factor que modifica el modo en que se procesa la información a nivel cognitivo ${ }^{27}$.

El estrés percibido difiere en función de los niveles de exposición al riesgo a nivel cualitativo y cuantitativo ${ }^{28}$, aunque en recientes estudios se ha tratado de "afinar" la medición de dichos niveles de exposición ${ }^{29}$. Los estudios muestran cómo la existencia de una alta percepción del riesgo se asocia a estrés postraumático y otras alteraciones relacionadas con el estrés, lo cual es consistente con estudios que han encontrado efectos directos entre la exposición a situaciones de combate y el desarrollo de estrés postraumático $28,30,31$.

De otro lado, a nivel grupal una mayor exposición a situaciones de riesgo predice una mayor cohesión de unidad. Parece que situaciones de estrés pueden llevar a los sujetos a la búsqueda de apoyo social, lo que favorece la cohesión ${ }^{32}$. Por último, bajos o moderados niveles de estrés parecen favorecer la unión de los individuos en la búsqueda de una meta compartida, lo que favorece el sentido de unidad grupal ${ }^{33}$.

Esto parece apoyar la investigación relativa a cómo la cohesión de unidad o el apoyo social reduce el riesgo a desarrollar reacciones por estrés de combate o estrés postraumático ${ }^{30,34,35}$. Una mayor cohesión facilita el contacto y la comunicación entre los miembros del grupo, lo que favorece la creación de un sentido a las experiencias traumáticas. Se sabe que este proceso de asignación de sentido a las experiencias está asociado a una mayor salud psicológica y física ${ }^{36,37}$. El apoyo social por otro lado también favorece el establecimiento de un locus de control interno, ayudando a las personas a sentir confianza a la hora de disponer de las habilidades y los medios adecuados para hacer frente a esas situaciones problemáticas ${ }^{38}$.

Como factor grupal, el apoyo psicosocial de compañeros es un elemento determinante en la incidencia del estrés ${ }^{6-12}$. Asimismo se subraya la importancia de la confianza en los compañeros y en el mando como requisito para un adecuado liderazgo ${ }^{15,39-41}$, y de igual modo en la aproximación basada en la toma de sentido en contextos peligrosos ${ }^{42}$. Ésta influye en la disposición del subordinado para aceptar los intentos de influencia de un líder en contextos normalizados ${ }^{43}$. Los entornos operativos de alto riesgo potencian el desarrollo de esta rápida confianza ${ }^{44,45}$. Asimismo, es determinante la importancia de la gestión afectiva del líder, y la utilidad de la cohesión de grupo y un buen clima de equipo ${ }^{42}$.

El apoyo social y la cohesión por tanto, son variables determinantes a la hora de afrontar el estrés. Un adecuado liderazgo basado en la confianza y en la competencia del líder para gestionar adecuadamente un grupo militar minimizando los riesgos es esencial. Por lo tanto, los recursos psicosociales de los que dispone una persona como el apoyo social percibido y recibido, la autoeficacia, las capacidades de afrontamiento, la maleabilidad para la recuperación, la tolerancia a la frustración, la resistencia al sufrimiento, etc., serán determinantes del grado de afectación 
traumática y/o postraumática tras haber pasado por una experiencia crítica de exposición al riesgo.

En resumen, la modulación de dichas respuestas, haciéndolas eficaces o no en la reducción de los niveles de estrés, dependen de las estrategias de afrontamiento que el sujeto emplea de un modo activo para controlarlas. Diversas variables relacionadas con dicho afrontamiento incluyen; (a) la percepción de autoeficacia, (b) la experiencia previa en dicho contexto operativo, (c) la percepción de apoyo por parte del grupo operativo de pertenencia, (d) el ejercicio del liderazgo centrado en la competencia del líder para el manejo de tales situaciones, etc...

El equipo de abordaje puede tener unas características diferenciales con respecto al resto de personal militar en la dotación de un buque debido a tres motivos fundamentalmente teóricos. De un lado, son equipos previamente seleccionados por su capacidad y perfil de afrontamiento de situaciones de riesgo. De otro, han recibido un entrenamiento específico que les lleva a la asunción de riesgos de un modo adaptativo ${ }^{15}$. Finalmente, la propia exposición al riesgo, puede mejorar la adaptación al mismo, entre otros motivos, por mecanismos de habituación, reforzando la percepción de capacidad de afrontamiento y éxito en operaciones, y la reducción adaptativa de las respuestas de ansiedad frente a potenciales agresores.

En este punto, resulta de interés conocer si existe un perfil psicológico diferencial en los equipos de abordaje respecto de la dotación, así como la relación de éste con los niveles de estrés percibido. Es un hecho que dicho grupo operativo tiene una mayor exposición al riesgo, debido a la misión encomendada de abordar y registrar buques sospechosos, que debería estar asociada a un mayor nivel de estrés percibido. Éste, a su vez, se ve modulado por variables de afrontamiento susceptibles de cambio debido al propio proceso de exposición anteriormente comentado.

Visto lo anterior, parece razonable estudiar los niveles de estrés percibido en los grupos de abordaje y el resto de dotación, así como la existencia de distintos perfiles psicológicos (individuales y grupales) asociados a distintos niveles de exposición a fuentes de riesgo, y por ende, si la pertenencia a dichas unidades de élite se relaciona con perfiles de mayor resistencia al estrés. Sería esperable encontrar:

Hipótesis 1. El nivel de estrés medio del personal perteneciente al equipo de abordaje debe ser igual o menor que el del resto de dotación. Aun cuando haya mayor exposición al riesgo, también existirán mecanismos de afrontamiento más eficaces.

Hipótesis 2. Existe un perfil diferencial entre el grupo de abordaje y el resto de la dotación del buque (grupo control), a nivel psicológico en variables que son asociadas en la literatura a un mejor afrontamiento del estrés.

\section{MATERIAL Y MÉTODO}

\section{Participantes}

La muestra estuvo compuesta por 120 militares de la Armada Española, desplegada en aguas del Mediterráneo e integradas en la SNMG-2 en un contingente de la OTAN. El rango de edad de dicha muestra se ubicaba entre los 19 y 46 años, con una media de 29 años desviación típica de 6,391. En cuanto al sexo, el $80 \%$ eran hombres mientras que el $20 \%$ eran mujeres. En relación al empleo, el $80 \%$ pertenecía a la categoría de Tropa y Marinería, y el $20 \%$ a la de Suboficiales. La distribución por nivel de estudios del modo que sigue: 6,9\% Licenciatura o Diplomatura universitaria, 27\% BUP, Bachiller, COU o Selectividad, 4\% Ciclo Formativo Superior o FPII, 21,3\% Ciclo Formativo Grado Medio o FPI y 40,8\% Secundaria Obligatoria o Graduado Escolar, todos ellos castellano parlantes.

La muestra se considera representativa de la población general de procedencia (población militar de las FAS), obteniendo los mismos porcentajes en cuanto al sexo que la población de origen.

La participación en dicho estudio fue voluntaria y anónima, dando su consentimiento informado.

\section{Variables}

Las variables objeto de estudio son; (a) la pertenencia al equipo de abordaje, con dos niveles (Boarding, $\mathrm{n}=17$ y Dotación, $\mathrm{n}=103$ ), empleada para generar los grupos de comparación, (b) el nivel de estrés percibido y (c) las distintas variables que definen el perfil psicológico (ver apartado instrumentos) a nivel individual y grupal en una unidad militar:

- Nivel de liderazgo: Relaciones verticales, conflictividad de la unidad, confianza en los mandos, confianza en el jefe inmediato y apoyo institucional percibido.

- Nivel grupal: Confianza en el grupo, confianza en los medios materiales, desempeño de la unidad, relaciones horizontales y cohesión

- Nivel individual: Confianza en sí mismo, autoeficacia, satisfacción laboral, apoyo social, legitimidad de la intervención militar y preocupaciones personales.

Las variables extrañas de mayor relevancia fueron fundamentalmente las vinculadas con el momento de evaluación y el procedimiento de aplicación de la prueba. En ambos casos se optó por un control basado en una adecuada estandarización de la prueba y una aplicación en óptimas condiciones de homogeneidad. En el caso del momento temporal se optó por la "constancia" en la aplicación ${ }^{46,}$ de modo que en todos los casos se evaluó al personal en el tercer mes de navegación.

\section{Instrumentos}

\section{Datos sociodemográficos}

Se registraron las variables sociodemográficas correspondientes a la descripción de la muestra, así como para determinar la pertenencia al equipo de abordaje (boarding).

\section{Escala de estrés de Cohen}

Dicha escala fue originalmente desarrollada en 1883 por Cohen, Kamarck y Mermelstein ${ }^{47}$, y adaptada a nuestro país ${ }^{48}$ sobre población similar a la de nuestro estudio, se usa para medir el grado 
en el que los individuos perciben situaciones de su vida como estresantes. Los ítems evalúan el grado en el que la gente encuentra su vida impredecible, incontrolada o con sobrecarga. Todos estos aspectos han sido considerados como esenciales o ejes fundamentales en el concepto de estrés ${ }^{49-52}$. La prueba es un autoinforme que consta de diferentes ítems (según versión de la que existen diversas). En nuestro caso particular se utilizó una prueba consistente en 14 ítems a los que el sujeto debe contestar en función de la frecuencia en la que percibe los diversos aspectos recogidos en los mismos, con una escala graduada de 0 a 4 , donde 0 significa "Nunca" y 4 "Muy frecuentemente". Los ítems han sido balanceados en su forma de presentación (y en el sentido o dirección positiva o negativa que mantienen con el constructo que evalúan) para evitar efectos en la respuesta debidos al orden de los mismos y a las tendencias de respuesta (fenómeno de aquiescencia). Según la adaptación antes referida presenta una fiabilidad por medio del procedimiento de las dos mitades con corrección Spearman-Brown de 0,788.

\section{Cuestionario del Perfil de Moral de Unidad}

Esta prueba tiene como finalidad la evaluación del personal militar en diversas dimensiones ${ }^{53,54}$, recogidas anteriormente en el apartado variables, subapartado (c). La prueba, validada sobre una muestra de 8.311 militares españoles, consta de 162 ítems donde se evalúan diversos aspectos vinculados con el desempeño de las misiones encomendadas al personal militar. Dichos ítems se agrupan en tres grandes dimensiones relativas a aspectos individuales, de grupo y de liderazgo. A continuación se ofrece una definición de las distintas subdimensiones:

\section{Eje de liderazgo}

1. Relaciones verticales: Se define por las relaciones de los mandos con los subordinados en la unidad, el trato de respeto, el reconocimiento de sus méritos y desempeño laboral, y la atención recibida en cuestiones personales y relacionadas con el servicio. Define la ligazón social que se produce entre mandos y subordinados. Ejemplo de item: "Me siento apoyado por los mandos de mi unidad".

2. Conflictividad: Se define como una falta de vinculación, de confianza, unida al aumento del distanciamiento entre mandos y subordinados debido a estilos de liderazgo basados en la crítica, las acciones disciplinarias inadecuadas, la falta de reconocimiento y la presión y freno a la iniciativa de los subordinados. Ejemplo de item: "En mi unidad hay excesiva presión psicológica".

3. Confianza en los mandos: Se define por el nivel de confianza que tiene el soldado en sus mandos en relación a la eficacia que los mandos demuestran en sus cometidos, y por tanto su competencia profesional, así como la capacidad para motivar a los miembros de su unidad, junto con el nivel de cohesión que los mandos muestran entre sí. Ejemplo de ítem: "Confío en los mandos de mi unidad para ir a un conflicto bélico".

4. Confianza en el jefe inmediato: Se define por el nivel de competencia profesional percibido por parte del jefe in- mediato, la credibilidad general que inspira como fuente de información, y su capacidad para actuar como ejemplo frente a los soldados, lo cual contribuye positivamente en el desarrollo de la confianza y vinculación del subordinado con el mando de su unidad. Ejemplo de item: "Recibo un buen trato de mi jefe inmediato".

5. Apoyo institucional: Se define como el vínculo y compromiso social que se establece entre los miembros de la unidad y la institución militar, mediante los beneficios y prestaciones que la misma proporciona a través de sus mandos. Ejemplo de item: "Estoy descontento con las condiciones de vida en el Ejército” (ítem negativo)

\section{Eje grupal}

6. Confianza en el grupo: Se define como el nivel de ligazón social que se produce entre los miembros de la unidad a través de la confianza mutua que genera las experiencias de adiestramiento y preparación para el combate. Estas experiencias construyen la confianza en la propia unidad para afrontar un conflicto bélico y consecuentemente esto fortalecerá la cohesión horizontal de la misma. Ejemplo de item: "Mi unidad puede manejar con éxito situaciones militares imprevisibles”.

7. Medios materiales: Se define por el nivel de confianza de los miembros de una unidad en el tipo y las condiciones en las que se encuentra el armamento, los medios materiales y equipos de combate disponibles empleados en el desempeño de una operación militar. Ejemplo de item: "Me parecen fiables el armamento, materiales y equipos de mi unidad”.

8. Desempeño de la unidad: Se define como el nivel de moral, entusiasmo y satisfacción, buena organización, coordinación y trabajo en equipo que caracteriza una unidad. Asimismo incluye elementos vinculados con la claridad de rol, y el esfuerzo por el estricto cumplimiento del deber y las actividades asignadas a dicha unidad. Ejemplo de item: "Mi unidad, en conjunto, se esfuerza en ser la mejor".

9. Relaciones horizontales: Se define por la calidad de las relaciones que mantienen los distintos miembros de la unidad (compañeros) basadas en el apoyo y la preocupación mutua, la existencia de sentimientos de unidad, la cohesión, el espíritu de equipo y una buena calidad e intensidad de las relaciones interpersonales. Ejemplo de item: "Hay buen ambiente en mi unidad".

10. Cohesión: Se define por la existencia de intensos vínculos sociales entre los miembros, donde hay un proceso de identificación y compromiso con los valores, normas y metas colectivas, caracterizada por la existencia de sentimientos de unión y orgullo de pertenencia a la unidad. Ejemplo de item: "Mi unidad se merece mi lealtad".

\section{Eje individual}

11. Confianza en sí mismo: Se define por la autovaloración positiva que un soldado hace sobre sus conocimientos 
tácticos, su preparación militar, su nivel de aptitud física y su disposición psicológica para el afrontamiento de un conflicto bélico. Ejemplo de ítem: "Dudo de mi resistencia psicológica para afrontar un conflicto bélico" (item negativo).

12. Autoeficacia: Se define por la percepción que los individuos tienen en relación con la disposición de las capacidades y recursos necesarios para resolver situaciones imprevisibles, difíciles o amenazantes, y la influencia que dichas creencias positivas en tales capacidades y recursos tienen sobre la moral, la autoconfianza, y la disposición para el combate y el cumplimiento de la misión en operaciones. Ejemplo de item: "Puedo resolver cualquier problema si me esfuerzo lo necesario".

13. Satisfacción laboral: Se define por el nivel de motivación, entusiasmo y satisfacción con respecto a la utilidad y sentido de su trabajo en el Ejército. Ejemplo de item: "Me siento ilusionado con mi trabajo".

14. Apoyo social: Se define como el nivel de aceptación y apoyo percibido por el soldado por parte de su familia, su entorno social más cercano, y por la sociedad en general, así como la calidad de las relaciones establecidas entre su acuartelamiento y la localidad donde se ubica el mismo. Ejemplo de item: "Todos mis familiares aceptan que sea militar".

15. Legitimidad de la intervención militar: Se define como el grado en que el soldado considera legítima, adecuada o justificada una intervención militar en el extranjero por motivos políticos, humanitarios y de legítima de defensa, acorde a los acuerdos que los países mantienen sobre la seguridad y la defensa internacional. Ejemplo de item: "Es injustificable cualquier tipo de intervención militar en otro país" (item negativo).

16. Preocupaciones personales: Se define como el grado en el que los soldados se sienten preocupados por la situación personal, familiar o profesional en la que se encuentran debido a las vicisitudes asociadas a la vida militar. Ejemplo de item: "Estoy muy preocupado por mi futuro profesional".

El formato de respuesta consta de 5 opciones graduadas con puntuaciones de 1 a 5 , donde 1 significa "Totalmente en desacuerdo" y 5 "Totalmente de acuerdo". Asimismo los ítems han sido balanceados tanto en su presentación como en el sentido con respecto al constructo para evitar los fenómenos asociados al orden y transferencia, así como de aquiescencia. El valor de consistencia interna (alfa de Cronbach) es de 0,98 , con valores que oscilan entre escalas desde 0,69 a 0,92 .

\section{Procedimiento}

El procedimiento de investigación se basó en la recogida de datos para la evaluación de los parámetros y variables anteriormente comentadas, que se llevó a cabo por medio de instrumentos que cumplen con las garantías científicas de los test de evaluación psicológica en términos de fiabilidad y validez.

Se tomaron datos en una sola aplicación, para lo cual se informó al personal participante en la navegación acerca del de- sarrollo de la investigación, de los objetivos pretendidos y de la libre y voluntaria participación en la misma.

La aplicación del cuestionario se llevó a cabo en un solo momento, tras recibir autorización para el desarrollo de la investigación, así como el consentimiento informado y la voluntariedad de los participantes, que en ningún caso se negaron a la prueba, y que mostraron en todo momento un alto nivel de motivación e interés por la misma. Se citó a los participantes en la investigación en un lugar específico habilitado para la aplicación de la prueba que en este caso fue el comedor del buque.

Al objeto de evitar sesgos vinculados con el orden y transferencia en la aplicación de las pruebas, los modelos de cuadernillo empleados se diseñaron siguiendo un principio de "contrabalanceo"46 en la presentación de las pruebas, de modo que cada condición (prueba empleada; sociodemográfica, estrés, etc...) se presentara a cada sujeto un número igual de veces en la sesión de evaluación y cada condición precedería y seguiría a todas las otras condiciones un número igual de veces.

En relación al momento de evaluación, dado que el periodo de despliegue se prolongaba durante cinco meses, en todos los casos y a tenor de los cambios y evolución del estado psicológico que acontece en el personal militar desplegado en operaciones, tomamos decisiones sobre el momento que se consideraba más oportuno. Para ello se tuvo en cuenta la previsible variación de los parámetros evaluados a lo largo del tiempo conforme al modelo de los Ciclos Emocionales del Despliegue ${ }^{55}$, puesto que existe el tiempo suficiente para que se puedan producir oscilaciones en los diversos parámetros psicológicos, así como en los niveles de estrés percibido, influenciados por el momento de la navegación en el que nos encontremos.

Por ciclo emocional se considera el proceso de cambio psicológico (cognitivo, emocional y conductual), que se produce como consecuencia de la variación situacional a la que se enfrentan las tropas a lo largo de la navegación. Dada la información procedente del modelo sobre los ciclos emocionales y la influencia que sobre nuestras variables objeto de estudio es previsible, estimamos que el momento de evaluación óptimo se ubicaba en la fase de sostenimiento o recuperación y estabilización que acontece tras el primer mes y previo al último mes. En el caso de las misiones de cinco meses, por tanto, entre el segundo y cuarto mes. De este modo, se eligió la aplicación de la evaluación en torno al tercer mes de participación. Se considera éste el momento ideal ya que, según la literatura, encontramos una mayor estabilidad psicológica en los participantes, evitando por tanto la aparición de oscilaciones y/o "picos" no deseados en las variables objeto de estudio.

\section{Análisis}

Los datos obtenidos de la investigación fueron procesados informáticamente de un modo manual, e incorporados en una base de datos elaborada con el programa SPSS (Statistical Package for Social Sciences) en su versión 19. Asimismo, tales datos fueron procesados para su preparación a efectos de análisis.

Una vez depurados los casos con la eliminación de los casos que por la forma de responder al cuestionario hacían inviable su análisis, y era por tanto recomendable su eliminación, se proce- 
dió a realizar los distintos análisis estadísticos necesarios y enlazados con los objetivos de investigación perseguidos.

La presentación de resultados se hace conforme a las pautas de formato establecidas por la American Psychological Association (2009), $6^{\text {a }}$ edición ${ }^{56}$.

En los análisis empleados en los estudios de validación de la escala, se incluyeron fundamentalmente los siguientes tipos de análisis:

- Análisis de corte descriptivo y de frecuencias para variables de tipo sociodemográfico.

- Análisis comparativos para lo que se utilizaron pruebas de contraste basadas en el estadístico T de Student. Estadístico idóneo para la comparación donde los grupos poseen distinto tamaño muestral, prestando atención al principio de homocedasticidad.

- Para el cálculo del tamaño del efecto en las pruebas de significación estadística, se empleó el calculador online disponible en http://www.uccs.edu/ lbecker/, concretamente para la diferencia tipificada por medio de la d de Cohen, y para cuya interpretación se utilizan las normas ofrecidas por el mismo autor ${ }^{57}$

\section{RESULTADOS}

\section{Niveles de estrés percibido en el grupo de Boarding y la dotación del buque}

Se comprueba el cumplimiento de supuesto de aplicación de pruebas paramétricas. Se analizan los niveles medios de estrés percibido en ambos grupos. Se comprueba que los niveles medios son ligeramente superiores en el caso del equipo de Boarding (abordaje). Medias y desviaciones típicas en todos los grupos de comparación recogidas en la tabla 2.

Al utilizar el estadístico T de Student para comparar ambos grupos, los resultados no reflejan diferencias estadísticamente significativas en el caso del estrés percibido ${ }^{(1)}, \mathrm{t}(118)=0,528, \mathrm{p}$ $=.599, \mathrm{~d}=0.028,95 \%$ IC $[-.08, .13]$, Cohen's $\mathrm{d}=0.14$, de modo que el nivel medio de estrés en los sujetos que pertenecen al equipo de abordaje y los que pertenecen a la dotación no difieren significativamente a nivel estadístico.

\section{Diferencias psicológicas a distintos niveles entre el equipo de Boarding y la dotación del buque}

Al estudiar las diferencias entre el equipo de boarding y la dotación del buque, se analizan tres grandes niveles ofrecidos por el cuestionario CPMU, a saber; (a) liderazgo, (b) cohesión y (c) individual. El equipo de boarding posee unos niveles medios mayores que la dotación del buque. Esto significa que posee un mejor perfil de liderazgo, una mayor cohesión, y que sus miembros poseen un mejor perfil psicológico individual.

\footnotetext{
(1) Variable estrés percibido transformada por medio de la raíz de la variable, debido a la violación del supuesto de normalidad, para la aplicación de pruebas paramétricas (consultar tabla 2).
}

En relación a los factores correspondientes al nivel de liderazgo, resulta en todos ellos mayor la puntuación en el equipo de boarding que en la dotación, a excepción del factor relativo al "apoyo institucional".

Sin embargo, al utilizar el estadístico T de Student para comparar ambos grupos en los distintos factores o variables, solo encontramos diferencias estadísticamente significativas en el caso de la variable relaciones verticales, $\mathrm{t}(118)=2,1, \mathrm{p}=.038, \mathrm{~d}=0.5$, $95 \%$ IC $[.03,1]$, Cohen's $d=0.5$, de modo que los sujetos que pertenecen al equipo de boarding poseen relaciones verticales significativamente mejores, siendo el tamaño del efecto moderado (ver tabla 1 )

En el caso de las variables relativas a la cohesión, el nivel medio en todas ellas es asimismo superior en el equipo de boarding que en la dotación del buque, siguiendo la tendencia observada en el caso del liderazgo, salvo en el caso de la variable "medios materiales", donde parece existir una valoración peor que el resto de dotación.

No obstante, las siguientes variables muestran diferencias estadísticamente significativas (ver tabla 1):

- Confianza en el grupo, $\mathrm{t}(118)=2,24, \mathrm{p}=.027, \mathrm{~d}=0.43$, $95 \%$ IC $[.05, .8]$, Cohen's d $=0.53$, de modo que los sujetos que pertenecen al equipo de boarding poseen una confianza en el grupo significativamente mayor que la dotación, siendo el tamaño del efecto moderado.

- Desempeño de la unidad, $\mathrm{t}(118)=2,1, \mathrm{p}=.04, \mathrm{~d}=0.37$, $95 \%$ IC $[.73, .54]$, Cohen's d $=0.54$, de modo que los sujetos que pertenecen al equipo de boarding consideran que su unidad tiene un nivel de desempeño significativamente mayor que la dotación, siendo el tamaño del efecto moderado.

- Cohesión, $\mathrm{t}(118)=2,1, \mathrm{p}=.04, \mathrm{~d}=0.45,95 \%$ IC [.03, .86], Cohen's d $=0.58$, de modo que los sujetos que pertenecen al equipo de boarding perciben un nivel de cohesión significativamente mayor que la dotación, siendo el tamaño del efecto moderado.

- Relaciones horizontales, $\mathrm{t}(118)=2, \mathrm{p}=.048, \mathrm{~d}=0.42,95 \%$ IC $[.003, .83]$, Cohen's $\mathrm{d}=0.55$, de modo que los sujetos que pertenecen al equipo de boarding perciben unas relaciones horizontales (de grupo) significativamente mejores que la dotación, siendo el tamaño del efecto moderado.

Por último, se presentan las variables relativas a los factores individuales. En todas las variables igualmente el equipo de boarding presenta mejores puntuaciones que la dotación del buque.

La variable que presenta diferencias estadísticamente significativas es la confianza en sí mismo, $\mathrm{t}(118)=3, \mathrm{p}=.004, \mathrm{~d}=0.46$, $95 \%$ IC $[.15, .77]$, Cohen's $d=0.76$, de modo que los sujetos que pertenecen al equipo de boarding poseen una mayor confianza en sí mismos que los de la dotación, siendo el tamaño del efecto grande.

\section{DISCUSIÓN}

El presente trabajo de investigación muestra la creciente participación de las Fuerzas Armadas Españolas en diversos teatros de operaciones, desde el año 1992, y cómo dichos escenarios han 
Tabla 1. Comparación de medias en las distintas variables objeto de estudio entre el grupo de Boarding y de dotación del buque por medio del estadístico T de Student para muestras independientes

\begin{tabular}{|c|c|c|c|c|c|c|c|c|}
\hline \multirow[b]{3}{*}{ Estrés* } & \multicolumn{8}{|c|}{ Prueba $\mathrm{T}$ para la igualdad de medias } \\
\hline & \multirow{2}{*}{$\begin{array}{c}\mathrm{t} \\
, 528\end{array}$} & \multirow{2}{*}{$\begin{array}{c}\mathrm{gl} \\
118\end{array}$} & \multirow{2}{*}{$\begin{array}{c}\begin{array}{c}\text { Sig. } \\
\text { (bilateral) }\end{array} \\
, 599\end{array}$} & \multirow{2}{*}{$\begin{array}{r}\begin{array}{r}\text { Diferencia } \\
\text { de medias }\end{array} \\
, 02779\end{array}$} & \multirow{2}{*}{$\begin{array}{c}\begin{array}{c}\text { Error típ. de } \\
\text { la diferencia }\end{array} \\
, 05268\end{array}$} & \multicolumn{2}{|c|}{$\begin{array}{c}\text { 95\% IC para la } \\
\text { diferencia } \\
\text { Inferior Superior }\end{array}$} & \multirow{2}{*}{$\begin{array}{c}\begin{array}{c}\text { Tamaño } \\
\text { Efecto }\end{array} \\
0,14\end{array}$} \\
\hline & & & & & &,- 07653 & ,13212 & \\
\hline Relaciones verticales** & 2,099 & 118 &, 038 &, 50324 & ,23970 & 02858 & ,97791 & 0,50 \\
\hline Conflictividad & 1,186 & 118 & ,238 & ,21777 &, 18360 &,- 14582 &, 58135 & \\
\hline Confianza en mandos & 1,581 & 118 &, 117 & ,36499 & 23093 &,- 09231 &, 82230 & \\
\hline Confianza en el jefe & ,600 & 118 &, 550 & ,13618 & ,22712 &,- 31358 & ,58593 & \\
\hline Apoyo institucional & $-1,302$ & 118 & ,196 &,- 20186 & ,15506 &,- 50892 &, 10520 & \\
\hline Confianza en el grupo** & 2,240 & 118 &, 027 & ,42776 & , 19100 &, 04952 & ,80599 & 0,53 \\
\hline Medios materiales &,- 396 & 118 & ,693 &,- 07596 & ,19204 &,- 45625 & ,30434 & \\
\hline Desempeño unidad** & 2,096 & 118 &, 038 & ,37645 & , 17962 & ,02076 &, 73215 & 0,54 \\
\hline Cohesión** & 2,129 & 118 &, 035 & ,44803 & ,21044 &, 03130 &, 86476 & 0,58 \\
\hline Relaciones horizontales** & 1,998 & 118 &, 048 & ,41648 & ,20841 &, 00376 & ,82919 & 0,55 \\
\hline Autoeficacia & 1,154 & 118 & ,251 &, 16147 & ,13993 &,- 11563 & ,43856 & \\
\hline Confianza en sí mismo** & 2,973 & 118 &, 004 & ,45953 &, 15456 &, 15347 & ,76559 & 0,76 \\
\hline Moral satisfacción laboral & ,352 & 118 &, 725 &, 06689 & , 19002 &,- 30940 & ,44318 & \\
\hline Apoyo social &, 074 & 118 & ,941 &, 01123 &, 15200 &,- 28977 & ,31223 & \\
\hline Legitimidad de la guerra & 1,601 & 118 &, 112 & ,25328 &, 15820 &,- 05999 &, 56655 & \\
\hline Preocupaciones personales & 1,122 & 118 & ,264 & , 17797 & ,15865 &,- 13621 & ,49215 & \\
\hline
\end{tabular}

* La variable Estrés ha sido transformada a efectos de análisis estadístico

** Variables donde se hallaron diferencias estadísticamente significativas.

Tabla 2. Medias y desviaciones típicas en las variables de los grupos de comparación.

\begin{tabular}{|lcccc|}
\hline \multicolumn{2}{c}{ Abordaje } & \multicolumn{2}{c|}{ Dotación } \\
\hline & Media & $\begin{array}{c}\text { Desviacion } \\
\text { típica }\end{array}$ & Media & $\begin{array}{c}\text { Desviacion } \\
\text { típica }\end{array}$ \\
\hline Estrés percibido & 2,45 & 0,64 & 2,36 & 0,61 \\
Factor Liderazgo & 3,40 & 0,75 & 3,19 & 0,65 \\
Factor Cohesión & 3,49 & 0,64 & 3,18 & 0,65 \\
Factor Individual & 3,78 & 0,41 & 3,59 & 0,43 \\
Relaciones verticales & 3,56 & 1,09 & 3,06 & 0,88 \\
Conflictividad & 3,58 & 0,77 & 3,37 & 0,69 \\
Confianza en mandos & 3,39 & 1,03 & 3,03 & 0,86 \\
Confianza en el jefe & 3,69 & 1,03 & 3,56 & 0,84 \\
Apoyo institucional & 2,75 & 0,65 & 2,96 & 0,58 \\
Confianza en el grupo & 3,52 & 0,90 & 3,09 & 0,70 \\
Medios materiales & 3,05 & 0,84 & 3,13 & 0,71 \\
Moral desempeño & 3,52 & 0,67 & 3,15 & 0,69 \\
Cohesión & 3,65 & 0,75 & 3,20 & 0,81 \\
Relaciones horizontales & 3,73 & 0,74 & 3,31 & 0,80 \\
Autoeficacia & 4,09 & 0,53 & 3,92 & 0,53 \\
Autoconfianza & 3,97 & 0,66 & 3,51 & 0,58 \\
Satisfacción laboral & 3,76 & 0,81 & 3,70 & 0,71 \\
Apoyo social & 3,71 & 0,49 & 3,70 & 0,59 \\
Legitimidad de la guerra & 3,92 & 0,69 & 3,67 & 0,59 \\
Preocupaciones personales & 3,21 & 0,52 & 3,03 & 0,62 \\
\hline
\end{tabular}

cambiado especialmente a raíz de los atentados terroristas del 11 de septiembre de 2001 en Estados Unidos, donde la Seguridad y Defensa internacional ha puesto su atención en el terrorismo. La Armada Española participa en la operación antiterrorista Active Endeavour que supone el abordaje de buques con actividad sospechosa.

Este estudio trata de analizar las características diferenciales de los grupos especiales cuya misión es el abordaje de buques (boarding teams) con respecto al resto de dotación, en lo relativo a sus niveles de estrés percibido y el perfil psicológico individual y grupal que presentan.

Es un hecho que dichas unidades se enfrentan a riesgos reales de un modo frecuente, y que dicha exposición diferencial al riesgo puede tener un efecto en el nivel de estrés percibido por parte de estos grupos. No obstante, aun cuando los niveles de exposición al riesgo sean mayores, los niveles de estrés percibido no son significativamente distintos.

Según los resultados obtenidos en el presente trabajo, se confirma que el nivel de estrés percibido no difiere significativamente en el grupo de abordaje (boarding) con respecto 
al resto de dotación (no expuesto al estresor del abordaje), lo que sugiere que pueden existir variables que favorezcan las estrategias de afrontamiento por parte de dicho grupo, a la hora de gestionar adecuadamente dicho estrés, y que modifican positivamente la valoración que hacen del entorno operativo ${ }^{11}$.

La utilización de una prueba exhaustiva como el CPMU, permite el estudio de diversas variables de relevancia en relación al rol que cumplen en el afrontamiento de estresores según la literatura existente, con la que establecer un perfil psicológico individual y grupal de la unidad evaluada. Según los resultados, encontramos que el grupo de abordaje presenta un mayor nivel en todas las variables consideradas, a excepción del apoyo institucional y la confianza en los medios materiales. No obstante estas diferencias no son estadísticamente significativas.

El grupo de abordaje muestras diferencias significativas en la variable relaciones verticales del liderazgo, y en la confianza en sí mismo en los factores individuales. Es decir, según la definición de las variables, parece que las relaciones entre mandos y subordinados en el grupo de abordaje son mejores y se caracterizan por el respeto, el reconocimiento y la atención prestada. Asimismo, las relaciones son más estrechas y el vínculo más cercano. De otro lado, los miembros del grupo de abordaje poseen una autovaloración positiva en relación a sus conocimientos tácticos, de preparación militar y la disposición psicológica para afrontar los riesgos asociados a su misión.

Pero es en el nivel grupal, donde las diferencias entre el grupo de abordaje y el resto de la dotación se hacen más patentes (en cuatro variables de las cinco evaluadas), dado que se encuentran diferencias estadísticamente significativas en relación a la confianza en el grupo, el desempeño de la unidad, la cohesión y las relaciones horizontales. Estas variables evalúan diversos aspectos de la confianza en la capacidad de afrontamiento de dificultades del grupo, el entusiasmo, satisfacción, buena organización y coordinación del mismo, una adecuada calidad en las relaciones mantenidas por los distintos miembros de la unidad y un sentimiento de unión, identificación y compromiso con los valores, metas y normas de la unidad. En todos los casos los tamaños de los efectos encontrados fueron moderados, salvo en la confianza en sí mismo cuyo tamaño del efecto se considera grande, utilizando las normas de interpretación del estadístico ofrecidas por el autor ${ }^{57}$.

Estos resultados son coherentes con el hecho de que la incidencia del estrés depende de la cantidad de recursos psicosociales que se poseen o se creen poseer para afrontar las exigencias impuestas por determinadas situaciones ${ }^{6-12}$. Se observa de un modo especialmente destacado una superioridad en el grupo de abordaje en relación a la confianza y sentimiento de pertenencia e identificación con el grupo, lo que supone la primera fuente de apoyo social a la hora de afrontar situaciones de riesgo. En tales situaciones, hay una importante dependencia del compañero. Además, es un resultado coherente con la literatura debido a que a nivel grupal una mayor exposición a situaciones de riesgo predice una mayor cohesión de unidad, puesto que situaciones de estrés pueden llevar a los sujetos a la búsqueda de apoyo social, lo que favorece la cohesión ${ }^{32}$. Así, moderados niveles de estrés parecen favorecer la unificación de los individuos en la búsqueda de una meta compartida, lo que potencia el sentido de unidad grupal $^{33}$. Este hecho, es de gran importancia puesto que existen investigaciones que muestran cómo la cohesión de unidad o el apoyo social reduce el riesgo a desarrollar reacciones por estrés de combate o estrés postraumático $\mathrm{O}^{30,34,35}$, lo que está asociado a una reducción de bajas en el teatro de operaciones.

En este sentido, autores han hallado que el apoyo social favorece el establecimiento de un locus de control interno, ayudando a las personas a sentir confianza a la hora de disponer de las habilidades y los medios adecuados para hacer frente a esas situaciones problemáticas ${ }^{38}$, algo coherente con nuestro hallazgo de una mayor confianza en sí mismo. Por tanto se establece un mecanismo explicativo del incremento de la confianza en sí mismo basado en el locus de control interno favorecido por el apoyo social.

Finalmente, se han detectado mejores relaciones verticales con mandos, lo que es coherente con la necesidad de un adecuado liderazgo. Diversos autores resaltan su importancia ${ }^{15,39-41}$. La confianza emerge también como un importante requisito del liderazgo en la aproximación basada en la toma de sentido en contextos peligrosos ${ }^{42}$. Además, parece que los entornos operativos de alto riesgo, como puede ser el caso de las misiones de abordaje, potencian el desarrollo de esta rápida confianza ${ }^{44,45}$.

En resumen, los resultados muestran que el grupo de abordaje presenta un nivel medio de estrés percibido similar al de la dotación del buque, aun cuando tenga un mayor nivel de exposición al riesgo, debido a la misión que tiene encomendada de registro de buques sospechosos. Este hallazgo empírico, dado que en el concepto de estrés se pone el énfasis en la interpretación que el sujeto hace de la situación amenazante ${ }^{11}$, puede ser explicado en base al uso de estrategias de afrontamiento de mayor eficacia que las empleadas por el resto de la dotación. Al estudiar el perfil psicológico individual y grupal de dichos grupos hallamos una clara superioridad de los factores asociados a la cohesión de grupo y el apoyo social que sus miembros reciben del mismo. En segundo orden aparecen unas mejores relaciones con los mandos, así como una mayor confianza en sí mismo, que según algunos autores deriva del favorecimiento que el apoyo social tiene en el desarrollo de un locus de control interno, lo cual genera un sentimiento de control y eficacia a la hora de afrontar las misiones encomendadas.

Se han propuesto mecanismos explicativos basados en la selección de dichos grupos, así como en el entrenamiento, sin embargo, la literatura parece apuntar a que son las características intrínsecas de estos grupos, que, por medio de la exposición al riesgo, modifican su estructura interna en términos de relaciones entre sus miembros, favoreciendo así una mayor eficacia grupal, acometividad, y en último término, una mayor probabilidad de éxito a la hora de afrontar el estrés consecuente de las operaciones antiterroristas encomendadas.

Esta idea, sobre la importancia que tiene en un grupo militar operativo el apoyo grupal, y el sentimiento de respaldo generado por el compañero a la hora de compartir metas, y de enfatizar la convicción en la capacidad para afrontar las dificultades intrínsecas de las operaciones militares, es ya contemplada desde hace mucho tiempo, cuando el Coronel Ardant du Picq (mediados del siglo XIX) concluyó, de un estudio sobre el mantenimiento de soldados en combate, que; "cuatro hombres valientes que no se conocen entre ellos no se atreverán a atacar a un león, pero que otros cuatro hombres menos valerosos que se conozcan bien entre 
ellos, y que estén seguros de su confianza y de las consecuencias del apoyo mutuo atacarán de un modo eficaz" ${ }^{58,59}$.

Por tanto, este estudio muestra cómo los grupos de élite de la Armada poseen un perfil psicológico diferencial con respecto al resto de la dotación del buque en términos de cohesión, confianza en sí mismo y calidad de las relaciones entre mandos y subordinados dentro de un grupo de alta exposición a riesgos. Estas cualidades, según la literatura, probablemente son desarrolladas como consecuencia de la propia exposición a situaciones peligrosas $^{15}$, donde el compañero se vuelve una pieza clave en la supervivencia en los casos en los que sea previsible un enfrentamiento. Desde esta perspectiva la cercanía y la confianza en personas que son los únicos que en tales situaciones pueden darnos apoyo y favorecer nuestra seguridad, se convierte en el elemento clave en dicho perfil. De otro lado, se comprueba empíricamente que el nivel de estrés percibido no difiere del resto de la dotación, probablemente debido a dicho perfil adaptativo. Dada la relación que el estrés mantiene con diversos procesos desadaptativos (ya comentados; estrés de combate, estrés postraumático, procesos de enfermedad mediados por un deficiente nivel de inmunocompetencia, etc...), se constata que los grupos de élite poseen un afrontamiento óptimo de las situaciones de riesgo asociadas a su misión. Esta importancia que tiene la cohesión de grupo en la mejora del afrontamiento de situaciones de estrés, es ya conocida en el ámbito internacional, donde el Ejército de los Estados Unidos ha implantado programas como el COHORT para favorecerla ${ }^{60}$, por medio de una reducción de la rotación de efectivos en unidades, así como la exposición a duros programas de entrenamiento militar ${ }^{15}$, como mecanismo de incremento de ese nivel de cohesión y autoconfianza.

\section{CONCLUSIONES}

El grupo de abordaje más expuesto a situaciones de riesgo presenta niveles similares de estrés percibido en el desempeño sus misiones al del resto de dotación, probablemente debido a un mejor afrontamiento de tales situaciones. De otro lado se constata la existencia de diferencias estadísticamente significativas en el perfil de ambas poblaciones, destacando especialmente un mayor nivel de cohesión y apoyo social, seguido de unas mejores relaciones mando-subordinado y confianza en sí mismo. Dichas variables se han relacionado en la literatura con una mejor gestión del estrés reduciendo las posibles consecuencias psicopatológicas del mismo. La importancia de estas variables ha sido puesta de manifiesto en programas del Ejército Americano (COHORT).

Una mejora del estudio se basaría en la generalización a otras poblaciones de características similares en orden a determinar la posible réplica de tales hallazgos. Sería recomendable incrementar el tamaño de la muestra del grupo de abordaje, aspecto éste de difícil control por el investigador dada la propia composición del mismo. Es destacable la investigación sobre aspectos psicológicos percibidos tales como la vivencia subjetiva de estrés y la caracterización psicológica individual y grupal de los participantes, especialmente en contextos operativos donde, además de las limitaciones existentes en el diseño de investigación intrínsecas a la situación, existe poca literatura al respecto.

\section{BIBLIOGRAFIA}

1. Ortega, J. Texto extraído del artículo de Jorge Ortega: "Las operaciones en Bosnia Herzegovina (II)”. Publicación Atenea. 2009; 6: 48-55.

2. Bartone PT, Adler AB, Vaitkus MA. Dimensions in psychological stress in peacekeeping operations. Mil Med. 1998; 163: 587-593.

3. Castro C, Adler A. OPTEMPO: Effects on soldier and unit readiness. Parameters. 1999; 29: 86-95.

4. Bell DB, Bartone J, Bartone PT, Schumm WR, Gade PA. USAREUR family support during Operation Joint Endeavor: Summary report (ARI Special Rep. No. 34). Alexandria: U.S. Army Research Institute for the Behavioral and Social Sciences; 1997.

5. Campbell DJ, Hannah ST, Matthews MD. Leadership in Military and Other Dangerous Contexts: Introduction to the Special Topic Issue. Mil Psychol. 2010; 22(S1): S1-S14.

6. Cano-Vindel A. Consecuencias del estrés laboral. Madrid: Sociedad Española para el Estudio de la Ansiedad y el Estrés; 2002.

7. Cohen, S. Measures of psychological stress. [Internet]. San Francisco: UCSF Medical Center; 2000. [Actualizado feb 2000; citado abril 2013]. Disponible en: http://www.macses.ucsf.edu/research/psychosocial/stress.php

8. Lazarus RS. Theory-based stress measurement. Psychol Inq. 1990; 1: 3-13.

9. Lazarus RS. Progress on a cognitive-motivational-relational theory of emotion. Am Psychol. 1991; 46: 819-834.

10. Lazarus RS. Towar better research on stress and coping. Am Psychol. 2000; 55: 665-673.

11. Lazarus RS, Folkman S. Stress, appraisal, and coping. New York: Springer Publishing Company; 1984.

12. Lazarus R, Folkman S. Estrés y procesos cognitivos. Barcelona: Ediciones Martínez Roca; 1986.

13. Davies MNO, Underwood G. Cognition and stress. En Fink G, director. Encyclopedia of stress. vol. 1. San Diego: Academic; 2000. p. 478-483.

14. Monat A, Lazarus RS. Stress and coping -some current issues and controversies-. En Monat A, Lazarus RS, directores. Stress and coping: an anthology. 2a ed. Nueva York: Columbia University Press; 1985. p. 1-12.

15. Samuels S, Foster C, Lindsay D. Freefall, self-efficacy, and leading in dangerous contexts. Mil Psychol. 2010; 22: S117-S136.

16. Bandura A. Self-efficacy: Toward a unifying theory of behavioral change. Psychol Rev. 1977; 84(2): 191-215.

17. Bandura A. Self-efficacy: The exercise of control. New York: Freeman; 1997.

18. Hannah ST, Avolio B, Luthans F, Harms P. Leadership efficacy: Review and future directions. Leadersh Q. 2008; 19: 669-692.

19. Jex SM, Bliese PD. Efficacy beliefs as the moderator of the impact of work related stressors: A multilevel study. J Appl Psychol. 1999; 84: 349-361.

20. Jex SM, Bliese PD, Buzzell S, Primeau J. The impact of self-efficacy on stressor-strain relations: Coping style as an explanatory mechanism. J Appl Psychol. 2001; 86: 401-409.

21. Schaubroeck J, Merritt DE. Divergent effects of job control on coping with work stressors: The key role of self-efficacy. Acad Manage J. 1997; 40: 738-754.

22. Bliese PD, Stetz MC. Modeling the Effects of Efficacy, Justice, and Conflict Among Reservists Activated for Homeland Defense. Mil Psychol. 2007; 19(1): 27-43

23. Folger R, Cropanzano R. Organizational justice and human resources management. Thousand Oaks: Sage; 1998.

24. Smith HJ, Tyler TR, Huo YJ, Ortiz DJ, Lind EA. The self-relevant implications of the group-value model: Group membership, self-worth and treatment quality. J Exp Soc Psychol. 1998; 34: 470-493.

25. Hancock PA, Hoffman JE. Stress and cognitive workload. En Tactical display for soldiers: Human factors considerations. Washington DC: National Academy Press; 1997.

26. Mareth TR, Brooker AE. Combat stress reaction: A concept in evolution. Mil Med. 1982; 150: 186-190.

27. Harris WC, Hancock PA, Harris SC. Information Processing Changes Following Extended Stress. Mil Psychol. 2005; 17(2): 115-128.

28. Fontana A, Rosenheck R. Psychological benefits and liabilities of traumatic exposure in the war zone. J Trauma Stress. 1998; 3: 485-503.

29. Mitchell MM, Gallaway MS, Millikan A, Bell MR. Combat Stressors Predicting Perceived Stress Among Previously Deployed Soldiers. Mil Psychol. $2011 ; 23(6)$ : 573-586. 
30. Solomon Z, Garb R, Bleich A, Grupper D. Reactivation of combat-related posttraumatic stress disorder. Am J Psychiatry. 1987; 144: 51-55.

31. Solomon $\mathrm{Z}$. The impact of posttraumatic stress disorder in military situations. J Clin Psychiatry. 2001; 62(suppl. 17): 11-15.

32. Taylor SE, Klein LC, Gruenewald TL, Gurung RAR, Fernances-Taylor S. Affiliation, social support, and biobehavioral responses to stress. En Suls J, Wallston KA, editores. Social psychological foundations of health and illness. Malden: Blackwell Publishing; 2003. p. 273-277.

33. Fontana A, Rosenheck R, Horvath T. Social support and psychopathology in the war zone. J Nerv Ment Dis. 1997; 185(11): 675-681.

34. Brailey K, Vasterling JJ, Proctor SP, Constans JI, Friedman MJ. PTSD symptoms, life events, and unit cohesion in U.S. soldiers: Baseline findings from the Neurocognition Deployment Health Study. J Trauma Stress. 2007; 20: 1-8

35. Vogt DS, Samper RE, King DW, King LA, Martin JA. Deployment stressors and posttraumatic stress symptomatology: comparing active duty and National Guard/Reserve personnel from Gulf War I. J Trauma Stress. 2008; 21(1): 66-74

36. Baddeley JL, Pennebaker JW. Expressive writing. En O'Donohue WT, Fisher J, editores. General principles and empirically supported techniques of cognitive behavior therapy. Hoboken: John Wiley \& Sons; 2009. p. 295-299.

37. Pennebaker J. Opening up: The healing power of expressing emotions. New York: The Guilford Press; 1997.

38. Sarason IG, Sarason BR, Shearin EN, Peirce GR. A brief measure of social support: Practical and theoretical implications. J Soc Pers Relat. 1987; 4: 497-510.

39. Sweeney P. Do soldiers reevaluate trust in their leaders prior to combat operations? Mil Psychol. 2010; 22: S70-S88.

40. Fisher K, Hutchings K, Sarros J. The "bright" and "shadow" aspects of in extremis leadership. Mil Psychol. 2010; 22: S89-S116.

41. Olsen O, Eid J, Larsson G. Leadership and ethical justice behavior in a high moral intensity operational context. Mil Psychol. 2010; 22: S137-S156.

42. Baran B, Scott C. Organizing ambiguity: A grounded theory of leadership and sensemaking within dangerous contexts. Mil Psychol. 2010; 22: S42-S69.

43. Dirks K, Ferrin D. Trust in leadership: Meta-analytic findings and implications for research and practice. J Appl Psychol 2002; 87(4): 611-628.

44. Jarvenpaa SL, Leidner DE. Communication and trust in global virtual teams. Organ Sci. 1999; 10: 791-815.

45. Meyerson D, Weick K, Kramer R. Swift trust and temporary groups. En Kramer RM, Tyler TR, editores. Trust in organizations: Frontiers of theory and research. Thousand Oaks: Sage; 1996. p. 166-195.
46. Arnau, J. Fundamentos de psicología experimental. Vol 1. Barcelona: Facultad de Filosofía y Ciencias de la Educación de la Universidad de Barcelona; 1974.

47. Cohen S, Kamarck T, Mermelstein R. A global measure of perceived stress. J Health Soc Behav. 1983; 24: 386-396.

48. Trujillo HM, González-Cabrera JM. Propiedades psicométricas de la versión española de la Escala de Estrés Percibido (EEP). Psicol Conductual. 2007; 15: 457-477.

49. Averill JR. Personal control over aversive stimuli and its relationship to stress. Psychol Bull. 1973; 80: 286-303.

50. Cohen S. Environmental load and the allocation of attention. En Baum A, Singer JE, Valins S, editores. Advances in environmental psychology. Vol 1. Hillsdale: Erlbaum; 1978. p. 1-29.

51. Glass DC, Singer JE. Urban stress: Experiments on noise and social stressors. New York: Academic Press; 1972.

52. Lazarus RS. Psychological Stress and the Coping Process. New York: McGraw-Hill; 1966.

53. Trujillo HM, Piñeira L. Cuestionario de Perfil de la Moral de Unidad (CPMU). Granada: Dirección de Investigación y Análisis para el Combate, Mando de Adiestramiento y Doctrina del Ejército de Tierra; 2005.

54. Galindo J. Evaluación de la Moral Militar en zona de operaciones y su relación con parámetros adaptativos. Granada: Servicio de publicaciones de la Universidad de Granada; 2013.

55. Morse J. The new emotional cycles of deployment. San Diego: U.S. Department of Defense, Deployment Health and Family Readiness Library; 2006.

56. American Psychological Association. Publication Manual of the American Psychological Association. 6th ed. Washington DC: American Psychological Association; 2009.

57. Cohen J. Statistical power analysis for the behavioral sciences. 2nd ed. Hillsdale: Erlbaum; 1988.

58. Ingraham LH, Manning FJ. Cohesion: Who needs it, what is it and how do we get it to them? Mil Rev. 1981; June: 2-12.

59. Shay J. Preventing psychological injury in military service [Internet]. Boston: Department of Veteran Affairs Outpatient Clinic and Tufts Department of Psychiatry; 2000. [actualizado 24 May 2001; citado 1 abr 2013]. Disponible en: http://belisarius.com/umodern_business_strategy/shay/shay_prevent_ psy_injury.htm.

60. Vaitkus MA. Unit Manning System: Human dimensions field evaluation of the COHORT company replacement model (Walter Reed Army Institute of Research Tech. Rep. No. 94-0017). Fort Detrick: U.S. Army Medical Research and Materiel Command; 1994. 